\title{
La ecología alimentaria del pez endémico Girardinichthys multiradiatus (Cyprinidontiformes: Goodeidae), en el Parque Nacional Lagunas de Zempoala, México
}

\author{
Patricia Trujillo-Jiménez \& Efrén Espinosa de los Monteros Viveros \\ Universidad Autónoma del Estado de Morelos, Centro de Investigaciones Biológicas. Av. Universidad 1001 Col. \\ Chamilpa, C.P. 62210 Cuernavaca, Morelos, México; trujill@cib.uaem.mx
}

Recibido 11-III-2003. Corregido 09-IX-2005. Aceptado 12-V-2006.

\begin{abstract}
The feeding ecology of the endemic fish Girardinichthys multiradiatus (Cyprinidontiformes: Goodeidae) in Lagunas of Zempoala National Park, Mexico. Girardinichthys multiradiatus, locally known as "mexcalpique", is a small endemic fish of the Lerma river basin. Its presence in lakes (Zempoala) suggests a long-standing connection between these lakes and the river basin. The current range of this species in the Mexico and Toluca valley appears to have been reduced, making this park a refuge for the species. Nevertheless, little is known about its biology. We studied its diet and feeding habits in Acoyotongo Lake, Lagunas de Zempoala National Park $\left(19^{\circ} 01^{\prime} 30^{\prime \prime}-19^{\circ} 06^{\prime} \mathrm{N}, 99^{\circ} 16^{\prime} 20^{\prime \prime}-99^{\circ} 21^{\prime} \mathrm{W}\right)$ where seasonal collections were carried out. The gut contents of 97 specimens were identified to the most specific taxonomic category possible and analyzed with numeric and frequency of occurrence methods. The general diet of this species consists of twelve dietary components, eleven of which are of animal origin. Hymenopterans, springtails and chironomids represented the highest percentage of ingestion and preference. G. multiradiatus is as a carnivorous species with entomophagous tendencies. Rev. Biol. Trop. 54 (4): 1247-1255. Epub 2006 Dec. 15.
\end{abstract}

Key words: Goodeidae, Girardinichthys multiradiatus, Zempoala, feeding ecology, carnivorous.

Para los peces como sucede con todos los animales, es indispensable una nutrición adecuada para poder crecer y sobrevivir. A través de la observación en el campo, la identificación minuciosa de los contenidos del tracto digestivo y por los estudios fisiológicos en el laboratorio, los investigadores han aprendido acerca de los hábitos alimentarios, las clases de organismos que comen y los mecanismos que han desarrollado para realizar la digestión (Lagler 1977, Gerking 1994).

Gerking (1994) mencionó que para conocer la ecología alimentaria de los peces, es necesario saber de que se alimentan, hábitos alimentarios y en menor grado el área de forrajeo, asímismo, el conocimiento del alimento consumido por los peces puede ser importante para conocer la posición que dicha especie ocupa dentro de la cadena trófica y para saber cuál es el papel que desempeña en el ecosistema que se encuentran.

Uno de los conceptos más importantes que se maneja en la ecología alimentaria de los peces es el de la adaptabilidad trófica, que es una expresión que describe la conducta alimentaria de las especies, que consiste en la flexibilidad de cambiar de un tipo de alimento a otro cuando la ocasión surge. Werner y Hall (1976) mencionan que la dieta de los peces usualmente cambia durante su vida, principalmente debido a los cambios morfológicos acompañados del crecimiento, edad, uso del hábitat o por cambio en las tácticas de forrajeo. Werner y Gilliam (1984), y Holbrook y Schmitt (1988) citaron que los cambios en la dieta y hábitat podrían también ser medidos por la abundancia de las 
presas y el riesgo a la depredación y consecuentemente los efectos en las interacciones de las especies. De esta manera, para poder entender estos cambios es esencial realizar estudios de ecología alimentaria de los peces.

La familia Goodeidae en México está representada por aproximadamente 42 especies de peces vivíparos (Paulo-Maya y TrujilloJiménez 2000). Soto-Galera y Paulo (1995) mencionaron que la distribución actual de los goodeidos dista mucho de ser natural, ya que las modificaciones del hábitat, ocasionadas principalmente por la construcción de obras hidráulicas y la contaminación urbana, agrícola e industrial, en prácticamente todas las cuencas donde se distribuye esta familia, se refleja en la restricción de la distribución de la mayoría de estas especies.

En los lagos del Parque Nacional lagunas de Zempoala, Morelos, México se distribuyen poblaciones de Girardinichthys multiradiatus, conocido comúnmente como "mexcalpique" pez pequeño, vivíparo, endémico de la cuenca del río Lerma, considerado de interés especial, debido a su confinada distribución y abundancia. Se conoce poco de su biología y ecología como para elaborar estrategias de conservación de sus poblaciones o su domesticación para reproducirlo en cautiverio y repoblar los ecosistemas donde esté a punto de desaparecer. Con base en lo anterior con el presente estudio se pretende dar a conocer parte de la ecología alimentaria de $G$. multiradiatus de acuerdo a su dieta y hábitos alimentarios.

\section{MATERIALES Y MÉTODOS}

Área de estudio: El Parque Nacional Lagunas de Zempoala, pertenece al municipio de Huitzilac, Morelos y a los municipios de Ocuilán y Artega del Estado de México. Esta situado a $65 \mathrm{~km}$ al sur de la ciudad de México y a $38 \mathrm{~km}$ al norte de la ciudad de Cuernavaca (1901'30"'-1906' N, 99॰16’20"'-99²1' W) (Bonilla-Barbosa y Viana 1997). El presente estudio se realizó en el lago Acoyotongo o de Tlilac, se le conoce como el número cinco, se encuentra localizado a $1 \mathrm{~km}$ al noroeste del lago Zempoala (1903'43”' N y $99^{\circ} 19^{\prime}$ W), a una altitud de $2830 \mathrm{msnm}$, al pie de los cerros Los Alumbres y La Cachucha.

Método de estudio: Debido a que G. multiradiatus se clasifica como una especie rara en el presente estudio solamente se llevaron a cabo tres colectas, dos en la época de estiaje (febrero1999 y marzo2000) y una en la época de lluvias (octubre1999).

Las colectas se realizaron con una red de arrastre de $3 \mathrm{~m}$ de longitud y luz de malla de $9 \mathrm{~mm}$; los ejemplares capturados se fijaron con solución acuosa de formaldehído al $10 \%$ (Windell y Bowen 1978); a cada uno de los ejemplares se les tomaron datos morfométricos en milímetros tales como: longitud total (LT); longitud patrón (LP) para establecer clases de talla y el peso con aproximación a décimas de gramo. Los tractos digestivos se extrajeron y analizaron de acuerdo a los criterios de Lagler (1977) y Gerking (1994). La identificación del contenido estomacal se llevó a cabo hasta el taxón particular más posible, basándose en los criterios de Needham y Needham (1979), Pennak (1978), Bland (1979), Chu (1979) y MacCafferty (1983).

Para la cuantificación del contenido estomacal se aplicaron los métodos numérico por cuadrícula y Frecuencia de aparición; así mismo, se aplicó el Índice de Albertaine (1973), el cual establece una escala en la cual los valores de 0 a 0.10 ubican al componente alimenticio como accidental; de 0.11 a 0.50 son ocasionales o secundarios y de 0.51 a 1.0 son preferentes. Para evaluar la amplitud del espectro trófico se utilizó el índice de Levin en el cual no es indispensable la evaluación de la biomasa de los posibles componentes alimenticios en la naturaleza (Ludwing y Reynolds 1988). Para medir el traslape de nicho se utilizó el índice de MacArthur y Levin (1967), modificado por Pianka (Krebs 1999), el cual presenta un intervalo de 0 (recursos no usados en común) a 1 (traslape máximo). Se utilizó la prueba de $\chi^{2}$ para determinar si las diferencias existentes entre sexos y clases de talla son significativas. 


\section{RESULTADOS Y DISCUSIÓN}

Dieta general: Con el análisis del contenido estomacal de 97 ejemplares de G. multiradiatus, se encontró que la dieta general de esta especie consta de doce componentes alimenticios, de los cuales once son de origen animal, registrándose que la mayoría de ellos son grupos de insectos tales como Hymenoptera, Odonata, Ephemeroptera, Diptera, Thysanoptera, Hemiptera, Coleoptera y Collembola y un componente de origen vegetal representado por las algas filamentosas. Con base en lo anterior, la población de G. multiradiatus del Lago Acoyotongo del Parque Nacional Lagunas de Zempoala es considerada como carnívora especialista (entomófaga), lo cual es corroborado con el resultado del índice de amplitud de nicho de Levin, con el que se obtuvo un valor bajo (0.48) que de acuerdo a la escala dada por Levin (Ludwing y Reynolds 1988) se registra como especialización.

Soto-Galera y Paulo (1995) mencionan que Allotoca dugesii, presenta características semejantes a G. multiradiatus, dado que ingiere insectos, por lo que es considerada carnívora con tendencias insectívoras. Duarte (1981) y Godínez (1989) mencionan que Xenotoca variata se alimenta de diversos componentes alimenticios (vegetales, zooplancton, anfípodos, insectos, peces y materia orgánica); sin embargo, el grupo ingerido predominante es el de los insectos, con base en lo anterior, estos autores consideran a esta especie como omnívora con franca tendencia carnívora (entomofagía).

Dentro de la familia Goodeidae no todas las especies presentan la misma tendencia, registrándose que Goodea atrippinis es una especie omnívora con preferencia por los vegetales también en menor proporción otros grupos como insectos y rotíferos (Barragán y Magallón 1994). Ilyodon whitei, tiene una dieta variada, por lo que se le considera, eurifágico oportunista, exhibiendo una preferencia por los vegetales (Trujillo-Jiménez y Díaz-Pardo 1996).

Los resultados citados junto con lo mencionado por Wootton (1990), permiten inferir que los peces presentan gran plasticidad en su ecología alimentaria, debido a que tienen la capacidad de ocupar distintos niveles tróficos, que van desde las especies herbívoras hasta las carnívoras secundarias e incluso algunas especies aprovechan la materia orgánica en descomposición, es decir, utilizan el detritus como alimento.

Con base en el análisis de la dieta general de G. multiradiatus, se registró a los himenópteros y los colémbolos con los porcentajes más altos de ingestión, y de acuerdo con el método de frecuencia de aparición y el índice de Albertaine éstos son considerados como alimentos secundarios. Los dípteros (quironómidos y cobóridos) también exhibieron porcentajes relativamente altos y por lo tanto son estimados como alimentos secundarios. El resto de los componentes alimenticios de origen animal presentaron porcentajes relativamente bajos, contemplados por ésto como alimentos accidentales. En cuanto a los componentes de origen vegetal, se registraron algas filamentosas, las cuales mostraron un porcentaje relativamente bajo, y de acuerdo con los resultados obtenidos con el método de frecuencia y el índice de Albertaine, éstas son alimento secundario (Cuadro 1).

Los altos porcentajes de ingestión de himenópteros y colémbolos en $G$. multiradiatus, hacen suponer que se alimenta principalmente en la superficie del agua, debido a que los colémbolos presentan hábitos neustónicos y están asociados a las orillas y entre la vegetación acuática (McCafferty 1983). Los himenópteros son insectos terrestres, y su presencia en los contenidos estomacales sugiere que estos se acercan a la vegetación acuática en busca de alimento, reposo, etc., y por la actividad misma que exhiben caen al agua siendo ingeridos por los peces. Trujillo (1998) informó que H. bimaculata se alimenta principalmente de himenópteros en la parte superficial de la columna de agua del río Amacuzac, Morelos. Soto-Galera y Paulo (1995) informaron que A. dugesii se alimenta de insectos terrestres, incluyendo larvas, pupas y adultos de dípteros y coleópteros, señalando que esta especie busca su alimento en los diferentes niveles de la columna de agua. 
CUADRO 1

Dieta general de acuerdo con los métodos Numérico (No) y Frecuencia de Ocurrencia (FO)

TABLE 1

General diet using the Numerical method (No) and the Frequency of Occurrence method (FO)

Componentes Alimenticios

Hymenoptera

$24.47(42.14)$

Odonata Zygoptera

$2.63(6.06)$

Ephemeroptera Baetidae

$3.41(7.09)$

Diptera Chironomidae

$16.49(32.26)$

Diptera Chaoboridae

$12.14(21.52)$

Thysanoptera

$6.15(12.24)$

Hemiptera Corixidae

$3.14(9.15)$

Coleoptera Elmidae

$3.88(9.15)$

Collembola

$21.4(24.61)$

Cyclops sp.

$0.15(5.03)$

Osteichthyes

$0.8(4.12)$

Algas Filamentosas

\section{CUADRO 2}

Análisis de la dieta por sexos, de acuerdo con los métodos numérico (No) y Frecuencia de Ocurrencia (FO)

TABLE 2

Diet analysis of the sexes using the Numerical method (No) and the Frequency of Occurrence method (FO)

\begin{tabular}{lcc}
\multicolumn{1}{c}{ Componentes } & Hembras & Machos \\
Hymenoptera & $20.84(41.17)$ & $26.68(43.06)$ \\
Odonata Zygoptera & $4.72(8.82)$ & $1.74(4.96)$ \\
Ephemeroptera Baetidae & $6.6(8.82)$ & $1.94(6.55)$ \\
Diptera Chironomidae & $11.82(26.46)$ & $19.26(36.71)$ \\
Diptera Chaoboridae & $8.52(17.64)$ & $14.33(24.01)$ \\
Thysanoptera & $8.0(20.58)$ & $5.39(8.14)$ \\
Hemiptera Corixidae & $4.41(11.76)$ & $2.69(8.14)$ \\
Coleoptera Elmidae & & $4.02(11.31)$ \\
Collembola & $23.91(26.46)$ & $20.28(24.01)$ \\
Cyclops sp. & $0.42(8.82)$ & \\
Osteichthyes & $0.26(8.82)$ & $1.09(4.76)$ \\
Algas Filamentosas & $10.5(26.46)$ & $2.5(9.52)$
\end{tabular}

secundario en éstas, mientras que los machos presentaron porcentajes bajos (Cuadro 2).

El resultado obtenido con el índice de amplitud de nicho, registra que las hembras presentan una amplitud de nicho estrecha (0.015), mientras que los machos una amplitud de nicho relativamente alta (0.46). Zaret y Rand (1971) sugieren que un resultado del índice de traslape de nicho trófico de $0.60 \mathrm{o}$ mayor es significante, considerando ésto el traslape de la dieta por sexos de G. multiradiatus es alto (OIFN=0.92). Sin embargo, la prueba de $\chi^{2}$ demostró que no existen diferencias alimentarias entre sexos.

El análisis por sexos de G. multiradiatus estableció que no existen diferencias alimenticias, ya que los dos sexos comparten los diferentes componentes alimenticios. Lo mismo fue observado por Duarte (1981) quien analizó el contenido estomacal de ocho especies de la familia Goodeidae y de acuerdo con sus resultados ambos sexos se alimentan de los mismos componentes, $X$. variata (Godínez 1989) e I. whitei (Trujillo-Jiménez 
y Díaz-Pardo (1996) no presentan diferencias marcadas por sexo.

\section{Análisis de la dieta por clases de talla}

De acuerdo al contenido estomacal de la clase de talla I, se registró que los alimentos que exhibieron los mayores porcentajes de ingestión y preferencia fueron los himenópteros; los dípteros quironómidos; dípteros cobóridos y los colémbolos considerados por esto como alimentos secundarios, el resto de los componentes de origen animal presentaron porcentajes en ambos métodos relativamente bajos. En cuanto al alimento de origen vegetal (algas filamentosas), éstas registraron un porcentaje de ingestión bajo; sin embargo, su porcentaje de preferencia fue relativamente alto (Cuadro 3).

La dieta de la clase de talla II estuvo representada con los valores de ingestión y preferencia más altos por los himenópteros; dípteros quironómidos; dípteros cobóridos y colémbolos; excepto los quironómidos que son alimento preferente, el resto de los componentes de origen animal exhibieron porcentajes de ingestión y preferencia relativamente bajos, en cuanto a las algas, estas presentaron valores bajos (Cuadro 3). El resultado obtenido con el índice de amplitud de nicho, registra que ambas clases de talla presentaron una amplitud de nicho relativamente alta (0.43 I y 0.53 II). El índice de traslape entre las clases de talla I-II fue alto $(\mathrm{OIFN}=0.79)$. La prueba de $\chi^{2}$ demostró que no existen diferencias alimentarias entre ambas clases de talla $(\mathrm{P}>0.05)$.

Ross (1978); Livingston (1982) y Allan (1995) señalaron que algunos peces pueden ubicarse en un nivel trófico sin dificultad; en cambio, otros son más difíciles debido precisamente a la flexibilidad en los hábitos alimentarios y los cambios ontogénicos. Wootton (1990) informó que la dieta de los peces varía con el crecimiento, sin embargo, esto no siempre es así, como se puede observar en $G$. multiradiatus, que no presentó diferencias alimenticias en las clases de talla ya que ambas se inclinan principalmente por los himenópteros, dípteros quironómidos,
CUADRO 3

Análisis de la dieta por clases de talla, de acuerdo con los métodos Numérico (No) y Frecuencia de Ocurrencia $(F O)$

TABLE 3

Diet analysis classified by size using the Numerical method (No) and the Frequency of Occurrence method (FO)

Componentes

Alimenticios

Clase I Clase II

Hymenoptera

$30.24(47.85) \quad 15.05(38.03)$

Odonata Zygoptera

5.07 (11.94)

Ephemeroptera Baetidae

3.37 (6.42)

Diptera Chironomidae

12.05 (24.99)

$30.5(59.77)$

Diptera Chaoboridae

11.39 (22.14) $14.27(20.64)$

Thysanoptera

$6.09(12.14) \quad 5.87(11.94)$

Hemiptera Corixidae

$2.89(9.28)$

5.58 (11.94)

Coleoptera Elmidae

3.49 (7.85)

$6.62(16.29)$

Collembola

$24.26(26.42)$

15.14 (24.99)

Cyclops sp.

0.2 (4.99)

Osteichthyes

0.03 (4.99)

1.47 (8.69)

Algas Filamentosas

$5.92(19.28)$

$0.38(4.34)$

cobóridos y colémbolos. Toledo (1996) informó que la dieta de $P$. sphenops y $H$. bimaculata no tuvo variaciones significativas cuando se analizó con relación al crecimiento; puesto que tanto las tallas pequeñas como las grandes en ambas especies muestran preferencia por los mismos componentes alimenticios.

Existen otros estudios que corroboran lo mencionado por Wootton (1990) como el caso de Duarte (1981) quien menciona que G. atrippinis si presenta diferencias alimenticias por tallas, ya que esta especie exhibe preferencia por los vegetales en las primeras tallas y en las tallas grandes presenta una preferencia por los crustáceos. Trujillo-Jiménez y DíazPardo (1996), informaron que I. whitei muestra ciertas variaciones en cuanto a la cantidad y variedad del alimento ingerido, dado que las tallas pequeñas se inclinaron principalmente por alimento de origen animal, mientras que las intermedias y grandes prefirieron a los vegetales. Trujillo (1998) informó que el ciclído 
Cichlasoma nigrofasciatum en el río Amacuzac, Morelos, puede ser considerado como entomófago en la fase temprana de su vida, incidiendo principalmente sobre el simúlido Simulium pulvurulentum, y cuando alcanza su talla máxima se comporta como omnívoro.

\section{Análisis de la dieta en sexos por clases de talla}

Hembras: La dieta de las hembras de la clase de talla I, esta constituida por nueve componentes, registrándose a los himenópteros y a los colémbolos con los porcentajes de ingestión y preferencia más altos, seguidos de los dípteros quironómidos, cobóridos, tisanópteros con valores de ingestión relativamente bajos, no obstante, de acuerdo con los valores obtenidos con el método de frecuencia son considerados como alimentos secundarios, el resto de los componentes de origen animal, presentaron valores bajos por lo que son contemplados como alimentos accidentales. Las algas filamentosas exhibieron valores de ingestión y preferencia relativamente alto (Cuadro 4).

Seis componentes alimenticios constituyen la dieta de las hembras de la clase de talla II; observándose a los himenópteros, dípteros quironómidos y colémbolos con los porcentajes de ingestión y de preferencia más altos, considerados por esto como alimentos preferentes, excepto los colémbolos que fue alimento secundario, asímismo, los odonatos cigópteros presentaron valores de ingestión y preferencia relativamente altos, siendo por esto alimentos secundarios, mientras que los peces y las algas exhibieron porcentajes de ingestión bajos, sin embargo, por el resultado obtenido con el método de frecuencia son considerados como alimentos secundarios (Cuadro 4). El resultado obtenido con el índice de amplitud de nicho registró una amplitud de nicho relativamente alta (0.66 Talla I y 0.57 Talla II).

Machos: Los machos de la clase de talla I exhibieron diez artículos alimenticios dentro

CUADRO 4

Composición de la dieta en sexos por clase de talla, de acuerdo con los métodos Numérico (No) y Frecuencia de Ocurrencia (FO)

TABLE 4

Diet analysis of the sexes classified by size using the Numerical method (No) and the Frequency of Occurrence method (FO)

\begin{tabular}{lcccc}
\multicolumn{2}{c}{ Hembras } & \multicolumn{2}{c}{ Machos } \\
Componentes Alimenticios & Clase I & Clase II & Clase I & Clase II \\
Hymenoptera & $22.45(33.14)$ & $25.47(81.25)$ & $36.05(56.69)$ & $12.46(23.80)$ \\
Odonata Zygoptera & & $13.48(31.25)$ & & \\
Ephemeroptera Baetidae & $7.77(9.14)$ & & $1.29(5.47)$ & \\
Diptera Chironomidae & $9.95(17.14)$ & $26.19(68.75)$ & $11.54(27.43)$ & $30.77(51.58)$ \\
Diptera Chaoboridae & $10.19(17.14)$ & & $12.69(24.99)$ & $16.89(23.80)$ \\
Thysanoptera & $9.48(21.14)$ & & $3.67(5.47)$ & $11.71(18.25)$ \\
Hemiptera Corixidae & $4.59(9.14)$ & & $2.13(7.91)$ & $5.78(12.69)$ \\
Coleoptera Elmidae & & & $3.58(10.35)$ & $7.11(18.25)$ \\
Collembola & $24.34(25.14)$ & $32.65(43.75)$ & $25.63(27.43)$ & $13.82(23.80)$ \\
Cyclops sp. & $0.57(9.14)$ & & & $1.39(5.55)$ \\
Osteichthyes & & $1.09(12.5)$ & $0.06(2.43)$ & \\
Algas Filamentosas & $10.64(25.14)$ & $1.09(12.5)$ & $3.35(15.23)$ &
\end{tabular}


de su dieta, registrándose a los himenópteros colémbolos, dípteros quironómidos y cobóridos con los mayores porcentajes de ingestión y de preferencia, siendo considerados como alimento secundario, excepto los himenópteros que son alimento preferente, el resto de los componentes alimenticios de origen animal exhibieron porcentajes de ingestión y preferencia bajos, siendo contemplados como alimentos accidentales, las algas filamentosas mostraron un valor de ingestión bajo, sin embargo, de acuerdo con el porcentaje de preferencia son consideradas como alimento secundario (Cuadro 4).

La dieta de la clase de talla II estuvo constituida por ocho componentes alimenticios, de los cuales los dípteros quironómidos, dípteros cobóridos, colémbolos, himenópteros y tisanópteros fueron los que exhibieron los porcentajes mas altos de ingestión, asímismo, se registraron con los porcentajes de preferencia altos por lo que son considerados como alimento secundario, excepto los quironómidos que fue alimento preferente, el resto de los componentes alimenticios de origen animal presentaron valores relativamente bajos de ingestión, por lo que son considerados como alimentos secundarios o accidentales (Cuadro 4). El resultado obtenido con el índice de amplitud de nicho registraró una amplitud de nicho relativamente alta $(0.37$ Talla I y 0.65 Talla II).

Los resultados obtenidos entre hembras por clase de talla, informaron que no existen diferencias alimenticias entre las clases pequeña e intermedia, lo cual fue corroborado con el alto valor obtenido con el índice de traslape; sin embargo, los adultos registran ciertas variaciones alimenticias, pero posiblemente se deba al número bajo de ejemplares analizados. Los machos de las dos clases de talla no presentaron variaciones alimenticias, lo cual es corroborado por los altos valores obtenidos con el índice de traslape. Hess y Tarzwell (1942) y Daniels y Felley (1992) observaron que Gambusia affinis tiene diferencias alimenticias en las hembras por clases de talla, la cual se incrementó conforme ganaban talla, a diferencia del presente estudio en donde no existen diferencias.
De acuerdo con los resultados obtenidos con el índice de traslape de Levin en las diferentes combinaciones realizadas se observó traslapes significativos en todas las combinaciones: Sexos $(\mathrm{OIFN}=0.92)$, sexos por clases de talla (OIFN=0.68 I y $0.73 \mathrm{II})$, hembras por clases de talla $(\mathrm{OIFN}=0.80)$ y machos por clases de talla $(\mathrm{OIFN}=0.71)$. La prueba de $\chi^{2}$ demostró que no existen diferencias alimentarias entre sexos y clases de talla ( $\mathrm{p}>0.05)$.

Ross (1986) menciona que el uso de los recursos por los peces tiene gran influencia en las interacciones de las poblaciones y las comunidades, en la dinámica de la disponibilidad de los recursos y en el destino de los mismos en el ecosistema. Es por esto que los estudios del reparto de los recursos tienen como meta principal conocer los límites de la competencia intraespecífica e interespecífica. Así pues, la ausencia de marcadas discrepancias alimentarias sexuales en G. multiradiatus, probablemente se debe a que los recursos alimenticios son abundantes, que junto con el reparto de los recursos entre las diferentes tallas evita la competencia intraespecífica

\section{AGRADECIMENTOS}

Agradecemos a Héctor Quiroz Castelán, Isela Molina Astudillo, Juan Carlos Sandoval Manrique, Aquiles Argote Cortes y Topiltzin Contreras MacBeath, por la revisión crítica del manuscrito.

\section{RESUMEN}

Girardinichthys multiradiatus, conocido comúnmente como "mexcalpique" un pequeño pez endémico de la cuenca del río Lerma, cuya presencia en el parque Nacional Lagunas de Zempoala, sugiere una antigua conexión entre estos lagos y la cuenca. El área de distribución actual en la porción del valle de México y Toluca se ha visto reducida, por lo que este parque representa un refugio para la especie. Sin embargo, se conoce poco de la biología del mexcalpique. Presentamos datos sobre su dieta y hábitos alimentarios. El estudio se realizó en el Lago Acoyotongo del Parque Nacional Lagunas de Zempoala mediante muestreos estacionales. La identificación del contenido 
estomacal (97 ejemplares) se llevó hasta el taxón más específico posible. Para la cuantificación se utilizaron los métodos numérico y frecuencia de aparición. La dieta general de esta especie se encuentra constituida por doce componentes alimenticios, de los cuales once son de origen animal. Himenópteros, colémbolos y quironómidos fueron los que presentaron los mayores porcentajes de ingestión y preferencia. Esta es una especie carnívora con tendencias insectívoras.

Palabras clave: Goodeidae, Girardinichthys multiradiatus, Zempoala, ecología alimentaria, carnívoro.

\section{REFERENCIAS}

Albertaine, B.J. 1973. Biologie des stades juveniles de teleosteens Mugilidae Mugil auratus Rissso 1810, Mugil saliens Risso 1810. I Régimen alimentaire. Aquaculture 2: 251-266.

Allan, J.D. 1995. Stream ecology: structure and function of running waters. Chapman \& Hall, Londres, Inglaterra. $388 \mathrm{p}$.

Barragán, J. \& S.B. Magallón. 1994. Peces dulceacuícolas mexicanos. X. Goodea atrippinis (Cyprinodontiformes:Goodeidae). Zool. Inf. IPN 28: 27-36

Bland, R.G. 1979. How to know the immature insects. Brown, Iowa, EEUU. 409 p.

Bonilla-Barbosa, J. \& J.A.L. Viana. 1997. Listados florísticos de México. XIV: Parque Nacional Lagunas de Zempoala. Instituto de Biología. UNAM, México D.F., México. 31 p.

Chu, H.F. 1979. The immature insects. Brown, Iowa, EEUU. 61 p.

Daniels, L.G. \& J.D. Felley. 1992. Life history and foods of Gambusia affinis in two waterways of southwestern Louisiana. Southwest Nat. 37:157-165.

Duarte, S.P. 1981. Contribución al conocimiento de los hábitos alimentarios de doce especies de peces del lago Cuitzeo, Mich. Tesis de Licenciatura, Escuela Nacional de Ciencias Biológicas-IPN, México, DF., México. 162 p.

Gerking, D.S. 1994. Feeding ecology of fish. Academic, California, EEUU. 416 p.

Godínez, R.M.A. 1989. Aspectos biológicos y ecológicos de Xenotoca variata (Pisces:Goodeidae). Tesis de Licenciatura, Escuela Nacional de Ciencias Biológicas-IPN, México, DF., México. 50 p.
Hess, A.D. \& C.M. Tarzwell. 1942. The feeding habits of Gambusia affinis affinis, with special reference to the malaria mosquito, Anopheles quadrimaculatus. Am. J. Hyg. 35:142-151.

Holbrook, S.J. \& R.J. Schmitt. 1988. The combined effects of predation risk and food reward on patch selection. Ecology 69:125-134.

Krebs, J. CH. 1999. Ecological methodology. Cummings, Old Tappan, Nueva Jersey, EEUU. 620 p.

Lagler, K.F. 1977. Freshwater fishery biology. Brown, Iowa, EEUU. 421 p.

Livingston, R.J. 1982. Trophic organization of fishes in a coastal sea grass system. Mar. Ecol. Prog. Ser. $7: 1-12$.

Ludwing, J.A. \& J.F. Reynolds. 1988. Statistical ecology. Wiley, Nueva York, EEUU. 337 p.

MacArthur, R.H. \& R. Levin. 1967. The limiting similarity, converge, and divergence of coexisting species. Am. Nat. 101:377-385.

McCafferty, W.P. 1983. Aquatic Entomology. Jones and Bartlett, Boston, EEUU. 448 p.

Needham, J.G. \& P.R. Needham. 1979. Guía para el estudio de los seres vivos de las aguas dulces. Reverté, Barcelona, España. 82 p.

Paulo-Maya, J. \& P. Trujillo-Jiménez. 2000. Nueva especie de Ilyodon (Cyprinodontiformes:Goodeidae) de la cuenca del río Balsas, México. Rev. Biol. Trop. 48: 465-472.

Pennak, K. 1978. Freshwater invertebrate of the United States. Wiley, Nueva York, EEUU. 420 p.

Ross, S.T. 1978. Trophic ontogeny of the leopard searobin Prinotus scitulus (Pisces:Triglidae). Fish. Bull. US 76:225-234.

Ross, S.T. 1986. Resource partitioning in fish assemblages: A review of field studies. Copeia 2: 352-388.

Soto-Galera, E. \& J. M. Paulo. 1995. Peces dulceacuícolas Mexicanos. XI. Allotoca dugesii(Cyprinodontiformes: Goodeidae). Zool. Inf. IPN. 31: 5-15.

Toledo, B.H. 1996. Variación alimenticia de los poeciliidos Heterandria bimaculata y Poecilia sphenops en la presa Los Carros, Axochiapan, Morelos. Tesis de Licenciatura, Facultad de Ciencias Biológicas U.A.E.M., Morelos, México. 36 p.

Trujillo, J. P. 1998. Dinámica trófica de la ictiofauna del río Amacuzac, Morelos. Tesis de Maestría. Facultad de Ciencias, UNAM, México, DF. 101 p. 
Trujillo-Jiménez, P. \& E. Díaz-Pardo. 1996. Espectro trófico de Ilyodon whitei (Pisces:Goodeidae) en el río del Muerto, Morelos. Rev. Biol. Trop. 44:755-761.

Werner, E.E. \& F.J. Gilliam. 1984. The ontogenetic niche and species interactions in size-structured populations. Annu. Rev. Ecol. Syst. 15: 393-425.

Werner, E.E. \& D.J. Hall. 1976. Niche shifts in sunfishes: experimental evidence and significance. Science 191: 404-406.
Windell, J. T. \& S. H. Bowen. 1978. Study of fish diets based on analysis of stomach contents, p. 219-226. In T. Bagenal (ed.). Methods for assessment of fish production in freshwater. Blackwell, Oxford, Inglaterra.

Wootton, R.J. 1990. Ecology of teleost fishes. Chapman and Hall, Londres, Inglaterra. 404 p.

Zaret, T.M. \& A.S. Rand. 1971. Competition in tropical stream fishes: Support for the competitive exclusion principle. Ecology 52:336-346. 
